The contrast between the extent of breakage and the low level of digestion is the most surprising finding from the newly discovered coprolite. Not only does it throw new light on the ability of large carnivorous dinosaurs to break down the bones of their prey in their mouths, but it also tells us something about the physiology of digestion once the prey animal has been swallowed. All of that from a piece of dung!

Peter Andrews and Yolanda Fernandez-Jalvo are in the Department of Palaeontology, Natural
History Museum, London SW7 5BD, UK.

e-mail:P.Andrews@nhm.ac.uk

1. Chin, K., Tokaryk, T. T., Erickson, G. M. \& Calk, L. C. Nature 393, 680-682 (1998)

2. Gilette, D. D. \& Lockley, M. G. Dinosaur Tracks and Traces (Cambridge Univ. Press, 1989).

3. Kordos, L. Geol. Hungarica Palaeontol. 46, 257-415 (1985).

4. Leakey, M. D. \& Hay, R. L. Nature 278, 317-323 (1979).

5. Andrews, P. Owls, Caves and Fossils (Chicago Univ. Press, 1990).

6. Scott, L. Quat. Res. 28, 144-156 (1987).

7. Charig, A. J. \& Milner, A. C. Bull. Nat. Hist. Mus. Lond. (Geol.) 53, 11-70 (1997).

8. Johnson, C. R. Zool. J. Linn. Soc. 52, 315-336 (1973).

9. Fisher, D. C. Paleobiology 7, 262-275 (1981).

\title{
HIV
}

\section{Envelope's letters boxed into shape}

\section{John P. Moore and James Binley}

T he groups of Sodroski and Hendrickson have put their distinctive stamps on the HIV-1 envelope by delivering a package of information (on pages $648^{1}$ and $705^{2}$ of this issue, and in this week's Science $^{3}$ ) on the crystal structure of the gp120 surface glycoprotein. These findings complement reports ${ }^{4,5}$ on the structure of gp41, and have important implications for virology, immunology and vaccine development.

The two groups crystallized the 'core' of the gp120 molecule from the $\mathrm{HxBc} 2$ laboratory strain as a ternary complex with its primary receptor (CD4 domains 1 and 2) and an anti-gp120 antibody fragment called 17b, which partially mimics the HIV-1 co-receptor (CCR5/CXCR4). Although seemingly drastic, the modifications necessary to crystallize the gp120 core preserve its antigenic integrity $^{6}$, and they do not seriously affect the value of the structural information obtained.

Much of what has been surmised about the topology of gp120 by biochemical ${ }^{7}$, mutagenic $^{8,9}$ and immunochemical ${ }^{10}$ techniques is now confirmed by the crystal structure. Additional surprises are, however, nicely discussed by the authors ${ }^{1,2}$. These include the existence of a 'silent face' - a large, previously unsuspected surface — and the nature of the two-domain gp120 structure, which provides a natural mechanism for receptor-induced conformational changes. Residues from several regions of the gp120 core are brought together to form the broad area that associates with CD4 but, unexpectedly, many of the $\mathrm{CD} 4$ contacts are made using the peptide backbone of gp 120 amino acids, not their side chains. Because antibody epitopes usually involve side chains, this device allows HIV-1 to alter the residues that form the $\mathrm{CD} 4$ binding site, without penalty to receptor binding, while changing the antigenic structure of the site to evade receptor-blocking antibodies. Nonetheless, there are also critical contacts with a few conserved side chains, including a

'knob-and-socket' interaction involving the unusually protuberant Phe 43 of CD4 and a receptive hole in gp120. This, and other cavities revealed in the surface of gp120, provides a target for inhibitors of receptor binding.

The region of gp120 that binds the CXCR4 co-receptor is also revealed on the crystal structure, in surrogate, by the residues that contact (or are close to) the $17 \mathrm{~b}$ antibody fragment. These residues are located within the highly conserved stem of the V1/V2 structure, near the base of the V3

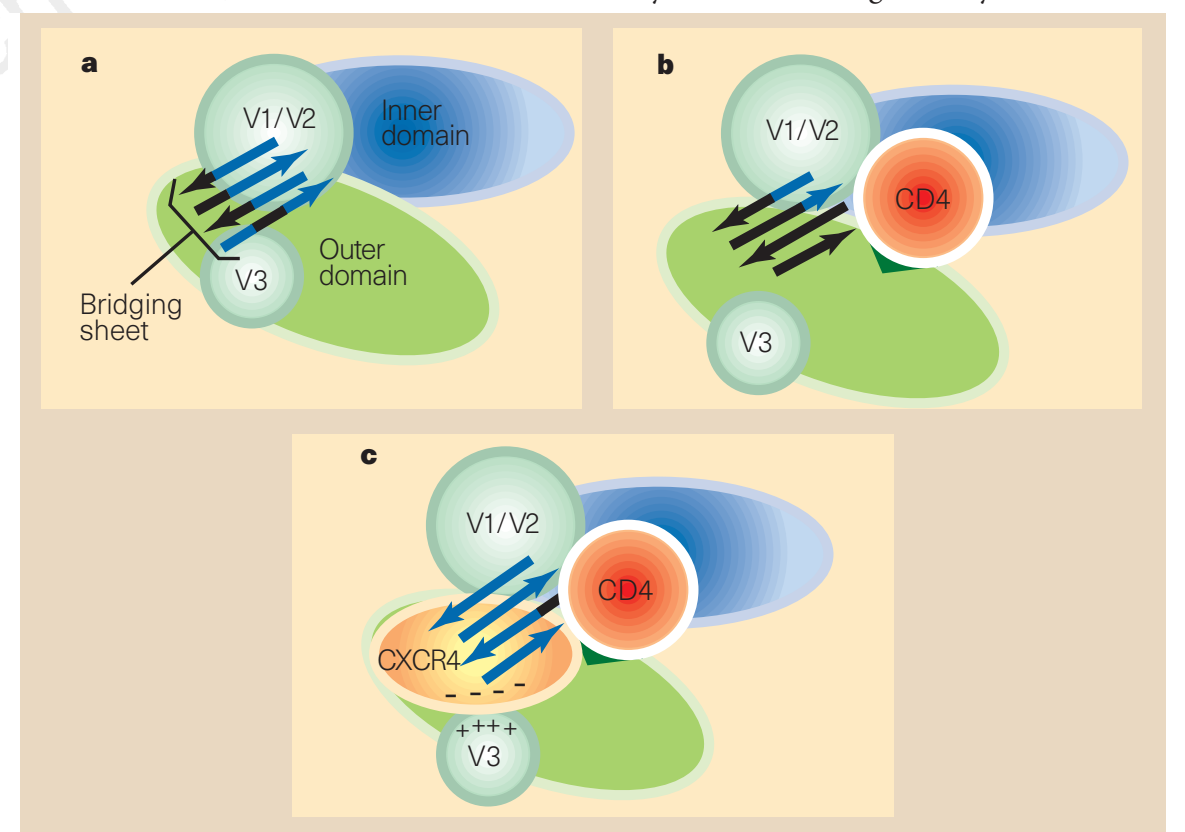

Figure 1 Proposed conformational changes during binding of gp120 to its receptor (CD4) and coreceptor (CXCR4/CCR5). These changes are based on the crystal structure of gp120 reported by Kwong et al. ${ }^{1}$, Wyatt et al. ${ }^{2}$ and Rizzuto et al. ${ }^{3}$. a, The bridging sheet (arrows), which spans the outer and inner gp120 domains, is made of residues in the stem of the V1/V2 loop and the CD4 region, and has not been shown to exist before binding to CD4. b, CD4 binds to a region that forms a crevice in gp120, triggering a conformational change that moves the V1/V2 loops away from the bridging sheet, and which may also move the inner and outer domains relative to one another, exposing the bridging sheet. $c$, The flexibility of $\mathrm{CD} 4$ allows the viral envelope to approach the target cell membrane, where the CXCR4 co-receptor interacts with the bridging sheet. Negatively charged regions of CXCR4 complement positive charges near the base of the V3 loop (this is speculative). The charge or conformation of the V3 loop may determine whether the CXCR4 or CCR5 co-receptor is used. 
site, occluding it in gp120s (such as $\mathrm{HxBc} 2$ ) that bind only CXCR4.

Importantly, creation or exposure of the highly conserved co-receptor-binding site requires that gp120 first binds CD4 (refs 11-13). This is another way for HIV-1 to evade humoral immunity - by the time the co-receptor site is ready to bind CCR 5 or CXCR4, the virus is already attached to CD4. Steric constraints will hinder access of antibodies to the co-receptor site under these conditions, explaining why primary isolates are poorly neutralized by the $17 \mathrm{~b}$ antibody ${ }^{2,3}$. The CD4-induced conformational changes in gp120 involve movement of the V1/V2 structure and, to a lesser extent, the V3 loop, away from the underlying co-receptor-binding site ${ }^{11}$. Although these variable loops are not present on the crystal structure, they have been modelled ${ }^{10}$ as a protuberance above the gp120 core. One way to view them is as an umbrella that shields the critical regions of gp120 from the rain of antibodies thrown at it by the humoral immune response; if a neutralizing antibody succeeds in binding to the variable loops, the virus will simply mutate the non-essential residues involved, and escape.

The virus has additional protection from humoral immunity by the extensive glycosylation of gp120. The authors ${ }^{1-3}$ modelled many of the glycans onto the crystal structure, clearly revealing how they shield receptor-binding regions of the peptide backbone from antibodies. This makes sense from the virus's perspective with rare exceptions, HIV-1 is neutralized by inhibition of its attachment to cellular receptors ${ }^{14}$. The same protective devices will also reduce the binding of gp120 to the immunoglobulin-like B-cell receptor, meaning that HIV-1 can also limit the production of neutralizing antibodies in the first place. Throw in observations that some strains of HIV-1 can even use anti-gp120 antibodies to increase their ability to fuse with host cells ${ }^{15}$ - presumably by occupying one of the three subunits of an assembled envelope glycoprotein trimer and inducing structural changes in the other two - and the war between HIV-1 and the humoral immune system takes an even more perverse twist.

The trimeric nature of the assembled gp120-gp41 complex can only be inferred from the crystal structure because the intersubunit contacts are between the gp41 moieties. But there is really only one way for all the components to fit together ${ }^{1,2}$. The immunogenicity and antibody reactivity of the assembled complex are even less than those of the gp120 monomer, perhaps because of steric considerations ${ }^{16,17}$, and this provides yet another level of protection the immune system is decoyed into making antibodies to disassembled gp120 that are poorly reactive, and hence ineffective, with virions. These protective measures may reduce HIV-1 infectivity in vivo, but they provide an overall advantage in the face of the immune response. In vitro, HIV-1 can afford to discard some of its protective armour, increasing its ability to bind receptors and infect its target cells at the (now irrelevant) expense of becoming neutralization sensitive ${ }^{18}$.

So what can be done to overcome the defences of HIV-1, given that an antibody response may be necessary to supplement vaccine-induced cellular immunity? There seems little to be gained by continuing to use simple gp120 subunits of whatever strain, alone or in combination. Antibodies elicited by such proteins play into the virus's hands because they attack its defences headon. If an arrow bounces off a tank, why use a quiver-full of the same design? Instead, we need to use the crystal structure to design a smart bomb with armour-piercing capacity, perhaps by modifying the antigenic structure of gp120. Already, there are indications that this may be possible. When glycosylation sites were deleted ${ }^{19}$ from the V1/V2 loops of the simian immunodeficiency virus gp120, not only was a neutralization-sensitive virus created, but the immunogenicity of the mutant virus was altered so that a better immune response was raised to the wild-type virus. Similarly, removing the V1/V2 loops from HIV-1 gp120 renders the conserved regions underneath more vulnerable to antibodies $^{11,20}$, although it is not yet known whether this will translate into improved immunogenicity. These and other approaches that will be stimulated by the new information on the structure of gp120 are part of the way ahead on the long road to developing an HIV-1 vaccine.

John P. Moore and James Binley are at the Aaron Diamond AIDS Research Center, The Rockefeller University, New York, New York 10021, USA.

\section{e-mail:jmoore@adarc.org}

1. Kwong, P. D. et al. Nature 393, 648-659 (1998).

2. Wyatt, R. et al. Nature 393, 705-711 (1998)

3. Rizzuto, C. et al. Science 280, 1949-1953 (1998).

4. Weissenhorn, W., Dessen, A., Harrison, S. C., Skehel, J. J. \& Wiley, D. C. Nature 387, 426-430 (1997)

5. Chan, D. C., Fass, D., Berger, J. M. \& Kim, P. S. Cell 89, 263-273 (1997).

6. Binley, J. M. et al. AIDS Res. Hum. Retroviruses 14, 191-198 (1998)

7. Leonard, C. K. et al. J. Biol. Chem. 265, 10373-10382 (1990)

8. Olshevsky, U. et al. J. Virol. 64, 5701-5707 (1990).

9. Helseth, E. et al. J. Virol. 65, 2119-2123 (1991).

10. Moore, J. P. \& Sodroski, J. J. Virol. 70, 1863-1872 (1996).

11. Wyatt, R. et al. J. Virol. 69, 5723-5733 (1995).

12. Wu, L. et al. Nature 384, 179-183 (1996).

13. Trkola, A. et al. Nature 384, 184-187 (1996).

14. Ugolini, S. et al. J. Exp. Med. 186, 1287-1298 (1997).

15. Sullivan, N. et al. J. Virol. (in the press).

16. Moore, J. P. et al. J. Virol. 69, 101-109 (1995).

17. Burton, D. R. \& Montefiori, D. AIDS 11, S587-S598 (1997).

18. Moore, J. P. \& Ho, D. D. AIDS 9, S117-S136 (1995).

19. Reitter, J. N., Means, R. E. \& Desrosiers, R. C. Nature Med. 4, 679-684 (1998).

20. Cao, J. et al. J. Virol. 71, 9808-9812 (1997).
Daedalus

\section{Thermal noise}

How to get rid of our mounting piles of organic waste? Oxidation is the obvious reaction for the job; but burning in air generates highly unpopular smoke. Waterbased oxidation would be far better. Sadly, it either needs ferocious reagents, such as fuming nitric acid, or extremely high temperatures and pressures, as in supercritical aqueous oxidation. Daedalus is looking for another way.

He notes that sonolysis, subjecting a reaction to intense high-frequency sound, can speed it up hundreds of times. The violent pressure-swings of the sound cause the liquid to cavitate, that is, to form tiny transient bubbles of vacuum. Their collapse produces vast temperatures and pressures; these create energetic free radicals which speed the reaction.

Sonolysis can certainly accelerate the oxidation of organics in solution. But Daedalus wants to destroy solids as well - old newspapers, plastic rubbish, food residues, discarded clothing, and the rest of our organic detritus. He points out that bubbles form easily on solid surfaces, especially irregular ones. Hence the 'boiling stones' used by chemists to aid smooth boiling, and the cavitation suffered by ships' propellers. A propeller can stir the water violently enough to cause cavitation; the bubbles form right on the metal where they can do the most damage.

In principle, therefore, a suspension of solid waste should oxidize if stirred with sufficient vigour - provided the waste itself was used as the stirrer. Now an object suspended in a conducting liquid threaded by a magnetic field experiences a force when a current is passed through the liquid: a sort of differential motor effect. So Daedalus will oxygenate his rubbish suspension, put it in a strong magnetic field, and pass high-frequency a.c. through it. The violent vibration of the solids against the surrounding water will cause cavitation at their interface. Bubbles will form and collapse on the solid surfaces, exactly where they are needed; the suspended waste will erode and oxidize rapidly.

Daedalus's waste-cavitation plant will suspend its shredded waste in air-saturated sea water, the cheapest oxidizing conducting solvent. The rubbish will simply fizz away to gas and ash, and the sterile effluent will be returned to the sea. The process should work on domestic sewage, too. Those lazy seaside towns that just pump the stuff out to sea will not even have to change their outfall pipe.

David Jones 